\title{
Revealing trade off relations among thermal coherences and correlations in Heisenberg XXX model under inhomogeneous magnetic field
}

\author{
Asad Ali \\ Department of Engineering Science Graduate School of Informatics and Engineering, \\ University of Electro Communications, Tokyo* \\ Mustansar Nadeem, A. H. Toor, Junaid-ul-Haq, and Shah Ahad \\ Department of Physics, Quaid-i-Azam University, Islamabad, Pakistan ${ }^{\dagger}$
}

We study the validity of quantum Fisher information (QFI) as a faithful quantum coherence and correlation quantifier by drawing a comparison with subsystem's coherence measure, first-order coherence (FOC) and the entanglement measure, Negativity to study the behavior of thermal quantum coherence and correlations in two qubit Heisenberg XXX model, placed in independently controllable magnetic field by systematically varying the coupling parameter, magnetic field and bath temperature for ferromagnetic and antiferromagnetic case. After carefully observing the profile of quantum coherence and correlation measures, we propose an inequality relations which shows that there may exist a quantitative relationship between QFI, Negativity and FOC in which, the equality exists at zero temperature. We identify QFI to be a more useful coherence quantifier, as it quantifies coherence of individual subsystems and correlations among the subsystems. On the other hand, FOC identifies coherence present in the individual subsystems only. A reciprocal relationship between Negativity and FOC is also observed in different cases. We also observe the existence of entanglement in ferromagnetic case, in contrast to simple Heisenberg XXX model in uniform magnetic field. We show that in the ferromagnetic case, a very small inhomogeneity in magnetic field is capable of producing large values of thermal entanglement. This shows that the behavior of entanglement in the ferromagnetic Heisenberg system is highly unstable against inhomogeneity of magnetic fields, which is inevitably present in any solid state realization of qubits. 
* asadalinorth@gmail.com

† mustansarqau@gmail.com 


\section{INTRODUCTION}

Quantum correlations and quantum coherence[1-2] are two essential aspects of quantum theory, and are extremely important for any quantum information or quantum computation, both in theory and in experiments. While the quantum correlations characterize the quantum features of a system with at least two parties, the quantum coherence is defined for a single as well as multipartite system [1]. Despite this difference, they are also intimately related to each other [3].

From the practical point of view, quantum correlations and quantum coherence are invaluable physical resources for implementing various quantum information and computation tasks, such as quantum key distribution (QKD) [4], super dense coding (SDC) [5], quantum cryptography (QC) [6], teleportation [7], and exponentially fast computing that requires entanglement [8]. A recently proposed quantum correlation measure called quantum discord (QD) [2] has been studied in several contexts with various operational interpretations and applications, such as quantum state merging (QSM) [9], remote state preparation (RSP) [10], and quantum metrology [11]. The ultimate quest for discovering a physically meaningful, and universal quantifier is a long-standing concern of theoretical physics. In search of finding universal resource quantifier for quantum correlation and coherence, the theorists have proposed many quantifiers, such as quantum Fisher information (QFI) [12], quantum discord (QD) [2], quantum coherence [1], quantum concurrence [8], first-order coherence (FOC) [13], Shannon-Jenson divergence coherence (SJDC) [14], and many more. The superiority of one quantifier over others in capturing quantum correlations and quantum coherence and their applicability, attracts researchers to investigate the dynamics of these quantifiers in different existing quantum mechanical systems, such as spin chains [15-20], optomechanics [21], quantum dots [22-23] and many other quantum states. These investigations are of great importance as they help us retrieve the quantum properties of a system and act as a tool for finding the universal quantum correlation and coherence quantifier.

Recently, some theoretical work on the trade off relations between entanglement and quantum coherence have been appeared in the literature as well [24-26]. Commonly used coherence measures consider a multipartite quantum system as a whole, omitting its internal mathematical structure and evolution in subsystems. The knowledge of the internal distribution of coherence between subsystems and their correlations becomes necessary for 
predicting the evolution (migration) of coherence in the studied system. Our focus here is to look at the behavior of FOC, Negativity and QFI in Heisenberg XXX model, as this model is an experimentally more feasible where the trade off relationship between FOC and Negativity can be seen and their connection with QFI is also discussed. In this paper, we collectively study the behavior of thermal FOC, QFI and Negativity in two-qubit Heisenberg's XXX model under inhomogeneous magnetic field. Two cases, ferromagnetic and antiferromagnetic spin chain are discussed for two-qubit under uniform as well as non-uniform magnetic fields.

The scheme of this paper runs as follows: In section 2 we discuss the two-qubit Heisenberg's XXX model under an inhomogeneous magnetic field. In section 3, we define FOC, QFI, Negativity and QM and present the 3D plots of each and quantifier in different cases. In section 4, we collectivelty discuss FOC, QFI, QM and Negativity for our model and discuss its different cases with the help of obtained 2D plots, and finally, in section 5, we summarized the conclusion with drawn results.

\section{THE MODEL}

First of all, let us briefly review the standard model of Heisenberg spin chain for our study. Considering two consecutive neighbor interactions, the Hamiltonian for a one-dimensional chain in an $n$ spin $\frac{1}{2}$ system can be written as;

$$
H=\frac{1}{2} \sum_{k=1}^{n}\left(J_{x} \sigma_{k}^{x} \sigma_{k+1}^{x}+J_{y} \sigma_{k}^{y} \sigma_{k+1}^{y}+J_{z} \sigma_{k}^{z} \sigma_{k+1}^{z}\right) .
$$

where $\sigma_{k}^{\alpha}(\alpha=\{x, y, z\})$ is the local spin pauli operators in the Hilbert space of $k^{t h}$ qubit, which satisfy $\sigma_{n+1}=\sigma_{1}$. Here the parameters $J_{\alpha}$ are the real coupling constants for the spin interaction. For $J_{x}=J_{y}=J_{z}=J$ (isotropy), $J_{x}=J_{y} \neq J_{z}$ (partial anisotropy) and $J_{x} \neq J_{y} \neq J_{z}$ (anisotropy), the Heisenberg chains are named as $X X X, X X Z$ and $X Y Z$ models, respectively. In addition, there exist two special case Heisenberg models, viz. the $X Y$ model for which $J_{z}=0$ and the Ising model for which $J_{y}=J_{z}=0$. From these models, our model of intereset in this article is Heisenberg $X X X$ chain model (In order to handle the number of parameters, we have chose XXX instead of XYZ or XY) in the presence of an inhomogenous magnetic field $b$ along with the z-direction, the Hamiltonian of which can be written as 


$$
H_{X X X}=J\left(\sigma_{1}^{x} \sigma_{2}^{x}+\sigma_{1}^{y} \sigma_{2}^{y}+\sigma_{1}^{z} \sigma_{2}^{z}\right)+(B-b) \sigma_{1}^{z}+(B+b) \sigma_{2}^{z},
$$

In which, $J<0$ corresponds to the ferromagnetic chain case and $J>0$ to the antiferromagnetic one, and $b$ indicates the degree of inhomogeneity in uniform magnetic field $B$. The two independently controllable parametrized magnetic fields $(B-b)$ and $(B+b)$ are applied to two qubits respectively. We have also set $\hbar=k_{B}=1$ and note that we are working in the units in which $B, J$ and $b$ are dimensionless. Furthermore, for sake of convenience we will set $J=1$ for antiferromagnetic case whereas for ferromagnetic case we will assume $J=-1$ The Hamiltonian (2) in the standard basis $\{|00\rangle,|10\rangle,|01\rangle,|11\rangle\}$ can be mathematically expressed as

$$
H_{X X X}=\left(\begin{array}{cccc}
J-2 B & 0 & 0 & 0 \\
0 & -J+2 B & 2 J & 0 \\
0 & 2 J & -J-2 B & 0 \\
0 & 0 & 0 & J+2 B
\end{array}\right) .
$$

The spectrum of eigenvectors and corresponding eigenvalues to (3) are as follows

$$
\begin{gathered}
E_{0}=-2 B+J, \\
E_{1}=-J-\sqrt{J^{2}+b^{2}}, \\
E_{2}=-J+\sqrt{J^{2}+b^{2}}, \\
E_{3}=2 B+J, \\
\psi_{0}=|00\rangle, \\
\psi_{1}=N_{1}\left(a_{1}|01\rangle+|10\rangle\right), \\
\psi_{2}=N_{2}\left(a_{2}|01\rangle+|10\rangle,\right. \\
\psi_{3}=|11\rangle,
\end{gathered}
$$


where $|0\rangle$ and $|1\rangle$ denotes spin up and down states respectively and $a_{1}=\frac{-b-\sqrt{J^{2}+B^{2}}}{J}, a_{2}=$ $\frac{-b+\sqrt{J^{2}+B^{2}}}{J}, N_{1}=\frac{1}{\sqrt{a_{1}^{2}+1^{2}}}, N_{2}=\frac{1}{\sqrt{a_{2}^{2}+1^{2}}}$.

The expression for the thermal equillibrium state of a quantum system is given as

$$
\begin{aligned}
& \rho(T)=\frac{1}{Z}\left(\exp \left(\frac{2 B-J}{k T}\right)\left|\psi_{0}\right\rangle\left\langle\psi_{0}\left|+\exp \left(\frac{J+\sqrt{J^{2}+b^{2}}}{k T}\right)\right| \psi_{1}\right\rangle\left\langle\psi_{1}\right|\right. \\
& \left.+\exp \left(\frac{J-\sqrt{J^{2}+b^{2}}}{k T}\right)\left|\psi_{2}\right\rangle\left\langle\psi_{2}\left|+\exp \left(\frac{-2 B-J}{k T}\right)\right| \psi_{3}\right\rangle\left\langle\psi_{3}\right|\right),
\end{aligned}
$$

In which $Z=\operatorname{Tr}\left(\exp \left(-\frac{H}{k T}\right)\right)$ is the partition function of the system and $k$ is Boltzmann constant. The Partition function and density matrix for the current system can be written as

$$
Z=\exp \left(\frac{2 B-J}{k T}\right)+\exp \left(\frac{J+\sqrt{J^{2}+b^{2}}}{k T}\right)+\exp \left(\frac{J-\sqrt{J^{2}+b^{2}}}{k T}\right)+\exp \left(\frac{-2 B-J}{k T}\right) .
$$

The matrix representation of the (13) in standard basis $\{|00\rangle,|10\rangle,|01\rangle,|11\rangle\}$ can be written as

$$
\rho(T)=\left(\begin{array}{cccc}
\rho_{11} & 0 & 0 & 0 \\
0 & \rho_{22} & \rho_{23} & 0 \\
0 & \rho_{32} & \rho_{33} & 0 \\
0 & 0 & 0 & \rho_{44}
\end{array}\right),
$$

with matrix elements being

$$
\begin{gathered}
\rho_{11}=\exp \left(-\frac{E_{0}}{k T}\right) . \\
\rho_{22}=N_{1}^{2} a_{1}^{2} \exp \left(-E_{1} / k T\right)+N_{2}^{2} a_{2}^{2} \exp \left(-E_{2} / k T\right) . \\
\rho_{23}=\rho_{32}=N a_{1} \exp \left(-E_{1} / k T\right)+N_{2}^{2} a_{2} \exp \left(-E_{2} / k T\right) . \\
\rho_{33}=N_{1}^{2} \exp \left(-E_{1} / k T\right)+N_{2}^{2} \exp \left(-E_{2} / k T\right) . \\
\rho_{44}=\exp \left(-E_{3} / k T\right) .
\end{gathered}
$$

In the next section, we define three quantifiers such as QFI, FOC and Negativity. 


\section{QUANTUM CORRELATIONS AND COHERENCE}

Here, we briefly discuss QFI, FOC and Negativity for our model individually.

\section{A. First order coherence}

Another quantifier for coherence in optical coherence theory and condensed matter physics is the measure of FOC of bipartite states [13]. It is related to the purity of the individual subsystems of the combined bipartite system. FOC in a multipartite system can be defined as

$$
D_{i}=\sqrt{2 \operatorname{Tr}\left(\rho_{i}\right)^{2}-1}
$$

where $\{i=A, B\}$ denotes the $i^{t h}$ subsystem. Then, we can obtain the coherence measurement of different subsystems when they are considered independently. The amount of coherence present in the system $\rho_{A B}$ can be represented as

$$
F_{o}(\rho)=D_{F}^{2}=\frac{\left(D_{A}^{2}+D_{B}^{2}\right)}{2} .
$$

The state $\rho_{A B}$ can be transformed via a global unitary $U$ to get state $\rho_{A B}^{\prime}=U \rho_{A B} U^{\dagger}$ such that the FOC can vanish, and the two subsystems become strongly correlated. On the other hand, for certain unitary operations $U^{\dagger}$, the maximum FOC can be obtained, $D_{F_{\max }}^{2}=$ $\left(\lambda_{1}-\lambda_{4}\right)^{2}+\left(\lambda_{3}-\lambda_{2}\right)^{2}$, where, $\lambda_{i}^{\prime} s$ are the eigenvalues of the state $\rho_{A B}$ in the decreasing order [13]. Furthermore, $D_{F_{\max }}^{2}$ can be called the degree of available coherence since it represents the maximum FOC extracted under a global unitary transform.

Fig. 1 represents the the 3D plots of FOC as function of magnetic field and temperature in which Fig.1(a) represents uniform antiferromagnetic case, Fig.1(b) uniform ferromagnetic, Fig.1(c) non uniform antiferromagnetic and Fig. 1(d) represents non uniform ferromagnetic case respectively.

\section{B. Quantum Fisher information}

Now we outline the QFI [12] for a quantum system. For a general phase estimation situation, a quantum state evolves under the unitary transformation and can be expressed as

$$
\rho_{\theta}=\exp (i G \theta) \rho \exp (-i G \theta)
$$




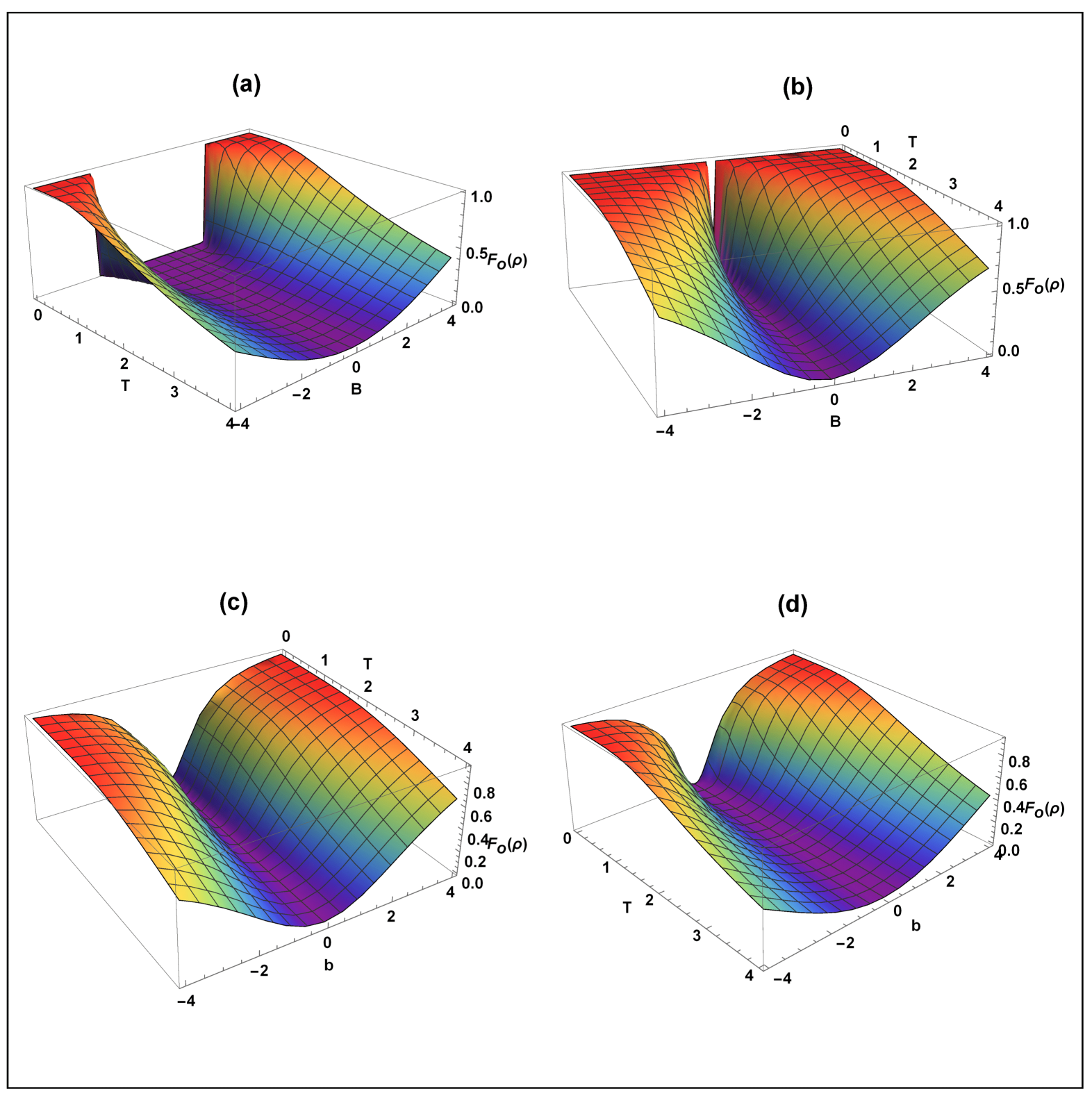

Figure 1. Surface plots of $F_{o}(\rho)$

Where $\theta$ is the parameter to be estimated and operator is $G$ is the generator. Correspondingly the accuracy of estimating $\theta$ is limited by Cramer-Rao inequality

$$
\triangle \theta \geq \frac{1}{\sqrt{\mu F\left(\rho_{\theta}\right)}}
$$

Where $\mu$ is the number of experiments and $F\left(\rho_{\theta}\right)$ is QFI. The QFI can be interpreted as the information on parameters encoded in the quantum state. Similarly, for an observer $O$ on a Hilbert space to be estimated, the QFI, i.e., the information involved in $\rho$ for observable $O$, 
can be given

$$
F(\rho, O)=\frac{1}{2} \operatorname{tr}\left(\rho L^{2}\right)
$$

Here $L$ is the symmetric logarithmic derivative determined by the following equality

$$
i[\rho, O]=\frac{1}{2}(L \rho+\rho L),
$$

where $[\rho, O]$ is a commutation relation If $\rho$ is a pure state, the QFI can be expressed as

$$
F(\rho, O)=\operatorname{tr}\left(\rho O^{2}\right)-[\operatorname{tr}(\rho O)]^{2}
$$

In the case of mixed states, the calculation of QFI becomes more complicated and will be shown in detail below. Generally, a mixed state $\rho$ can be described as

$$
\rho=\sum_{m} P_{m}|m\rangle\langle m|
$$

(20) where $|m\rangle$ and $P_{m}$ are respectively the eigenvectors and eigenvalues of the $m^{\text {th }}$ component of the state $\rho$. Exploiting (25) and (27) in (24) gives [27]

$$
F(\rho, O)=\sum_{m, n} \frac{\left(P_{m}-P_{n}\right)}{2\left(P_{m}+P_{n}\right)}|\langle m|O| n\rangle|^{2}
$$

Here $m$ and $n$ mark respectively the eigen-parameters $\left(|m\rangle,\left|P_{m}\right\rangle\right.$ and $|n\rangle$ and $\left|P_{n}\right\rangle$. We take a two-component composite system as an example and discuss the QFI calculation in detail. Suppose that $\rho_{i j}$ is any bipartite state while $A_{u}$ and $B_{u}$ are respectively arbitrary local orthonormal observable bases for two subsystems in $\rho_{i j}$. In this case, the QFI encoded in this two-component system with respect to the observables can be evaluated as [27],

$$
F(\rho)=\prod_{u} F\left(\rho_{i j}, A_{u} \otimes I_{j}+I_{i} \otimes B_{u}\right)
$$

which is also called the global information of $\rho_{i j}$. In general, for a two-qubit system, the local ortho-normal observables $A_{u}$ and $B_{u}$ can be taken as:

$$
A_{u}=B_{u}=\frac{I}{\sqrt{2}}, \frac{\sigma_{1}}{\sqrt{2}}, \frac{\sigma_{2}}{\sqrt{2}}, \frac{\sigma_{3}}{\sqrt{2}} .
$$

Here, $\sigma_{i}$ are the Pauli matrices for which $i=1,2,3$. Consequently, if $\rho_{i j}$ is given, the QFI can be calculated according to

Fig. 2 represents the the 3D plots of QFI as function of magnetic field and temperature in which Fig.2(a) represents uniform antiferromagnetic case, Fig.2(b) uniform ferromagnetic, Fig.2(c) non uniform antiferromagnetic and Fig. 2(d) represents non uniform ferromagnetic case respectively. 


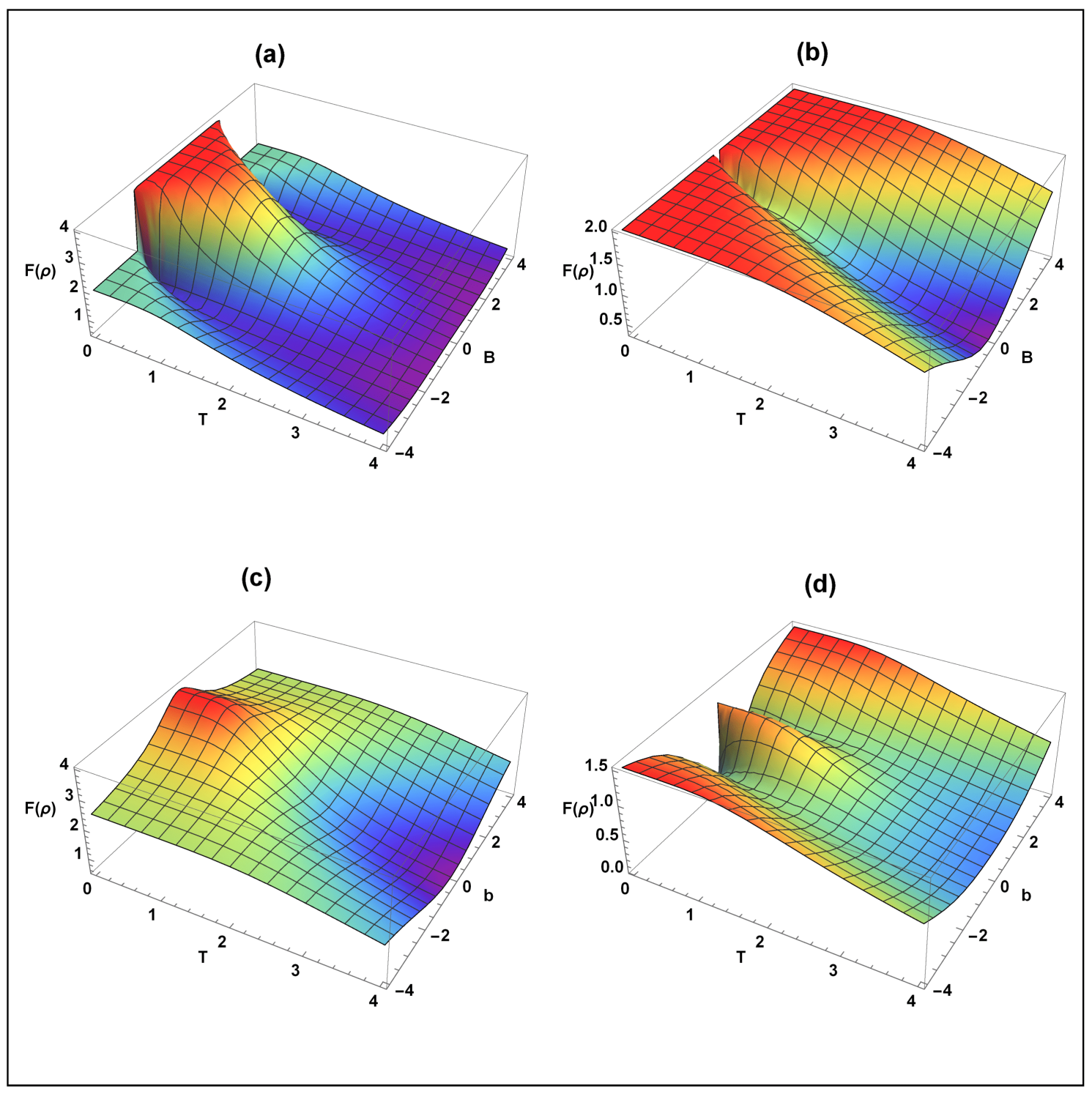

Figure 2. Surface plots of $F(\rho)$

\section{Quantum Mixedness}

For any arbitrary state $\rho$, the state is a pure state if

$$
\operatorname{Tr}\left(\rho^{2}\right)=1
$$

and if the state is mixed then

$$
\operatorname{Tr}\left(\rho^{2}\right)<1
$$

Thus the QM for any arbitrary state $\rho$ is defined as [28]; 


$$
M(\rho)=\frac{d}{d-1}\left(1-\operatorname{Tr}\left(\rho^{2}\right)\right)
$$

Where $d$ is the dimension of $\rho$ and $0 \leq M(\rho) \leq 1$.

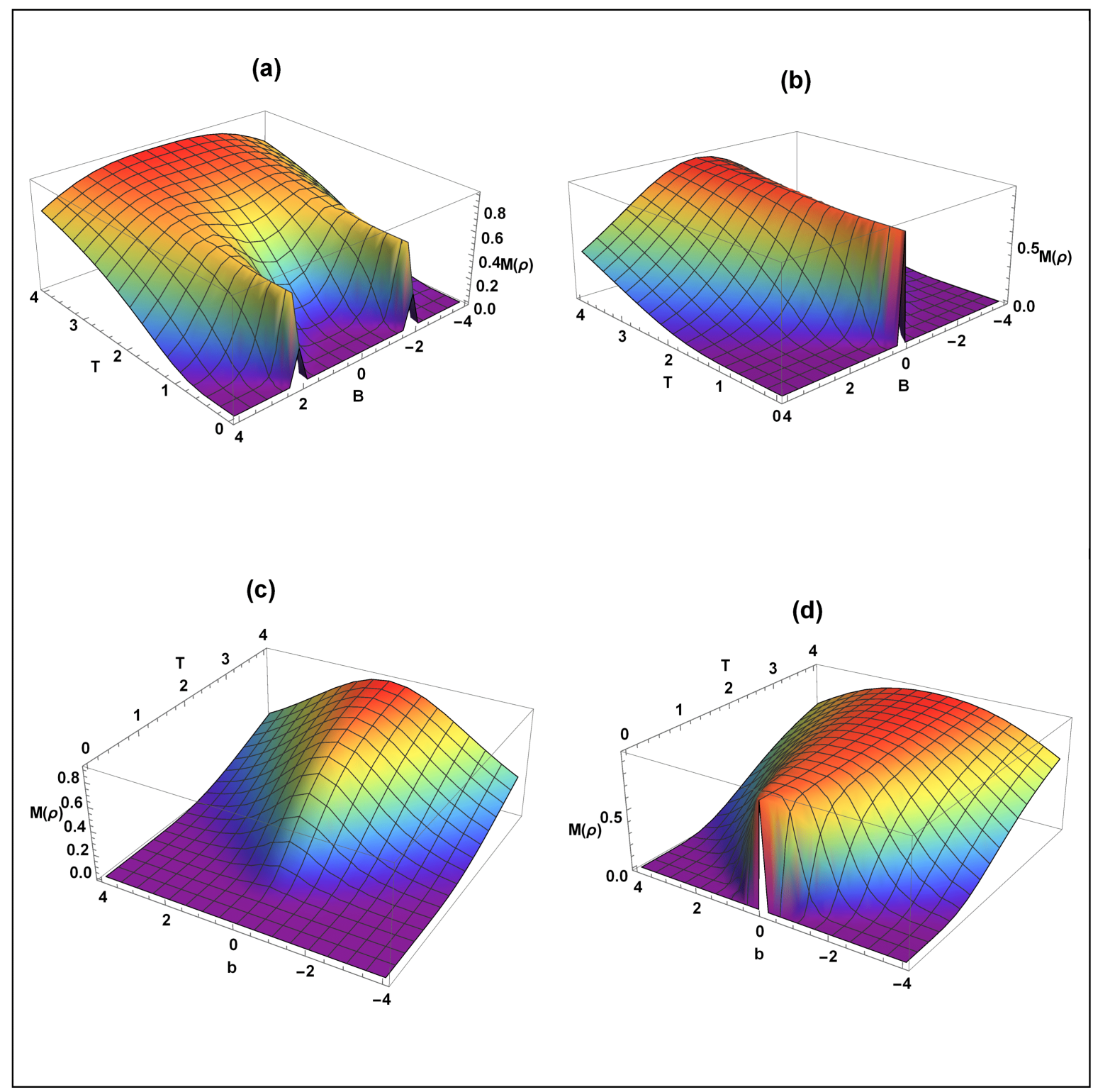

Figure 3. Surface plots of $M(\rho)$

Fig. 3 represents the the 3D plots of $\mathrm{QM}$ as function of magnetic field and $T$ in which Fig.3(a) represents uniform antiferromagnetic case, Fig.3(b) uniform ferromagnetic, Fig. 3(c) non uniform antiferromagnetic and Fig. 3(d) represents non uniform ferromagnetic case respectively. 


\section{Negativity}

Negativity in quantum information theory is a good measure of quantum entanglement because it is directly derived from PPT criterion for separability and is easy to compute [29]. It is proved to be an entanglement monotone and so is a proper measure of entanglement. For any density matrix $\rho$, the Negativity of subsystem is defined as

$$
N(\rho)=\frac{\left\|\rho^{T_{A}}\right\|-1}{2}
$$

Here, $\rho^{T_{A}}$ represents partial transpose of $\rho$ with respect to subsystem $A$ and

$$
\left\|\rho^{T_{A}}\right\|=\operatorname{Tr}\left(\rho^{T_{A}}\right)=\operatorname{Tr} \sqrt{\rho^{T_{A} \dagger}} \rho^{T_{A}}
$$

Fig. 4 represents the 3D plots of Negativity as function of magnetic field and temperature in which Fig. 4(a) represents uniform antiferromagnetic case, Fig. 4(b) uniform ferromagnetic, Fig. $4(\mathrm{c})$ non uniform antiferromagnetic and Fig. $4(\mathrm{~d})$ represents non uniform ferromagnetic case respectively.

\section{NUMERICAL RESULTS AND DISCUSSION}

In this section we report the collectively behavior of QFI, FOC and Negativity for four cases, two cases for antiferromagnetic and other two for ferromagnetic scenario through 2D plots.

\section{A. Uniform antiferromagnetic case}

The collective plots of QFI, FOC, QM and Negativity as a function of $B$ at different fixed values of the temperature $T$ are plotted when $J=+1$ and $b=0$ (except for $\mathbf{F i g}$. 5 (a) where $B=b=0)$ are shown in Fig. 5 .

At $T=0$, (see Fig. 5(a)) with increase in $B$ both in $+x$ and $-x$ axis, there is a critical point at $B=B_{c}=2$ at which Negativity, QFI and FOC become a non-analytic function of $B$ which gives the signature of phase transition. Here Negativity and QFI both are maximum for $|B|<\left|B_{c}\right|$ and are minimum for $|B|>\left|B_{c}\right|$, when $T=0$. This means for $|B|<\left|B_{c}\right|$ at $T=0$, the ground state of system is in entangled state, whereas for $|B|>\left|B_{c}\right|$ it is not entangled state. 


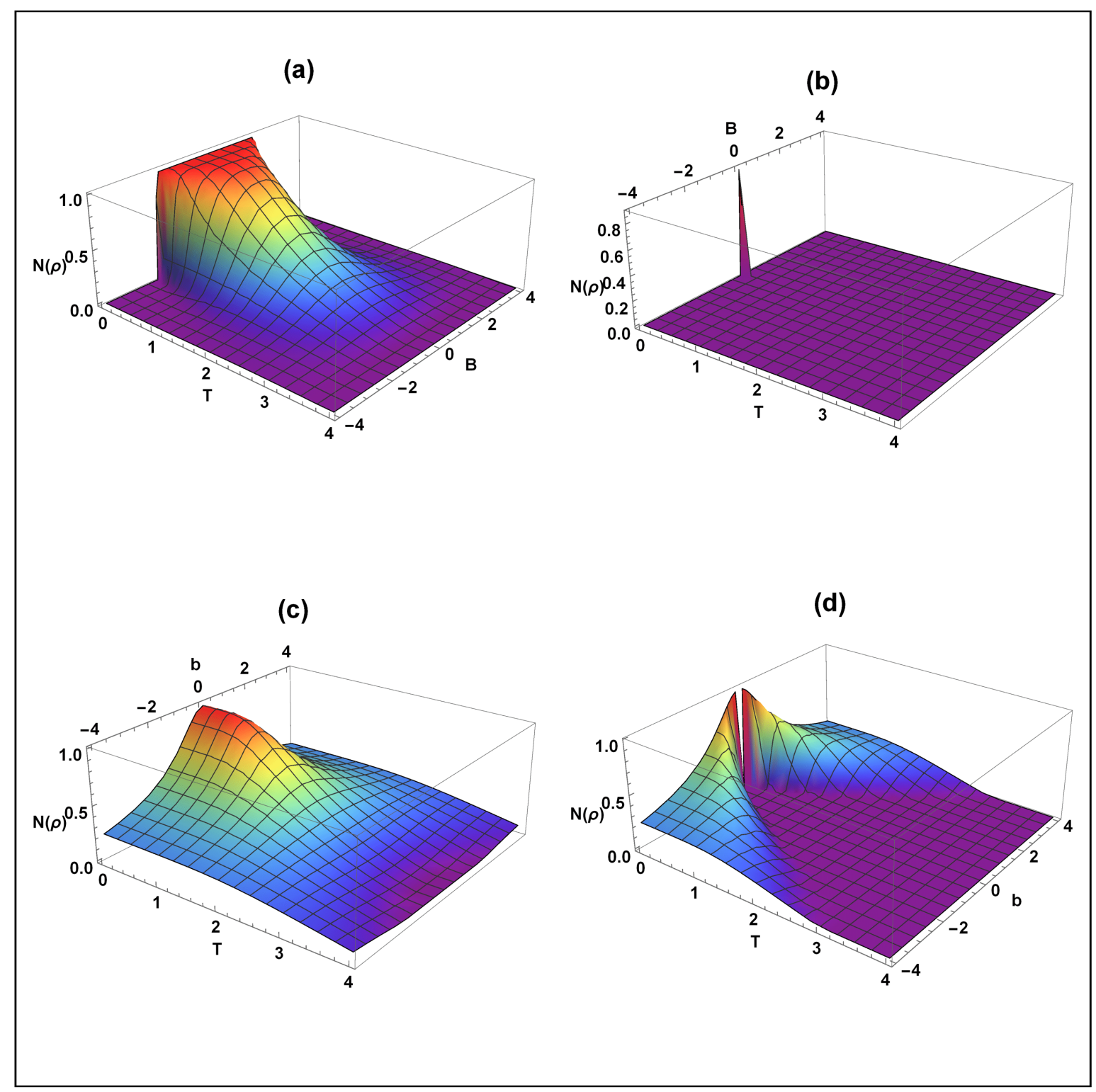

Figure 4. Surface plots of $N(\rho)$

Furthermore, for larger values of $T$ (Fig. $5(\mathrm{~b}), \mathbf{5}(\mathrm{c}), \mathbf{5}(\mathrm{d}))$, the peak of value of Negativity decreases and completely decrease to zero for larger values of $B$ beyond the critical magnetic field $\left|B_{c}\right|=2$. The value of the Negativity is trivially to be zero for large $T$ for an arbitrary value of $B$. This is because thermal state approaches to be the completely mixed state for $T$ getting infinity, and there are a separable ball with a strictly positive volume about the completely mixed state in an arbitary finite bipartite dimensional Hilbert space.

While observing from Fig. 5(a) to Fig. 5(d), it is obvious that Negativity has maximum 
value $N(\rho)=1$ at $T=0$ and $N(\rho)<1$ for $T>0$ which is due to the fact that at $T=0$, the ground state is in maximally entangled state and with the Increase in $T$ entanglement is lost due to mixing of entangled ground state with its unentangled states.the ground state of system is in entangled state, whereas for $|B|>\left|B_{c}\right|$ it is not an entangled state. It is very interesting to note that the QFI behaves in the same as Negativity in the range where $|B|<\left|B_{c}\right|$ where FOC is zero whereas In the range $|B|>\left|B_{c}\right|$, QFI shows the same behavior as FOC where Negativity is zero from which we observe that there exists some kind of trade off behavior between entanglement and subsystems coherence which is very prominent for small $T$ values whereas for large $T$ values, this effect cannot be seen because entanglement is not robust and decays with $T$. So we realize QFI as a faithful quantifier which captures both entanglement and hidden coherence of individual spins but it is unable to differentiate between FOC and Negativity against $B$. It is also evident from the same Fig. $\mathbf{5}$ at $T=0$ the system is in perfectly pure state that is why QM is zero and with rise in $T$ the peak value of QM increases whereas it can be suppressed by increasing $B$.

After carefully looking at the plots, we propose an inequality which shows that there may exist a quantitative relationship between QFI, FOC and Negativity as a function of both uniform and non uniform magnetic field for different fixed value of $T$;

$$
2(1+N(\rho)) \leq F(\rho)
$$

Similarly,

$$
\begin{gathered}
3+\left(N(\rho)-F_{o}(\rho)\right) \leq F(\rho) \\
F_{o}(\rho)+N(\rho) \leq 1
\end{gathered}
$$

In these relations equality holds at $T=0$.

These set of inequalities works fairly well in anitferromagnetic case when observed against magnetic field both uniform and non uniform except for ferromagnetic non uniform mangetic field case. Fig. 6 shows the plot of QFI, Negativity and FOC as a function of $T$ at different fixed values of $B$ when $J=+1$. For $B=0$ in Fig. $6(\mathbf{a})$, it is shown that the ground state is maximally entangled and so Negativity $N(\rho)=1$. Whereas for different fixed values of $B>0$, peak value of $N(\rho)<1$ which shows that the role of $B$ and $T$ are to diminish 


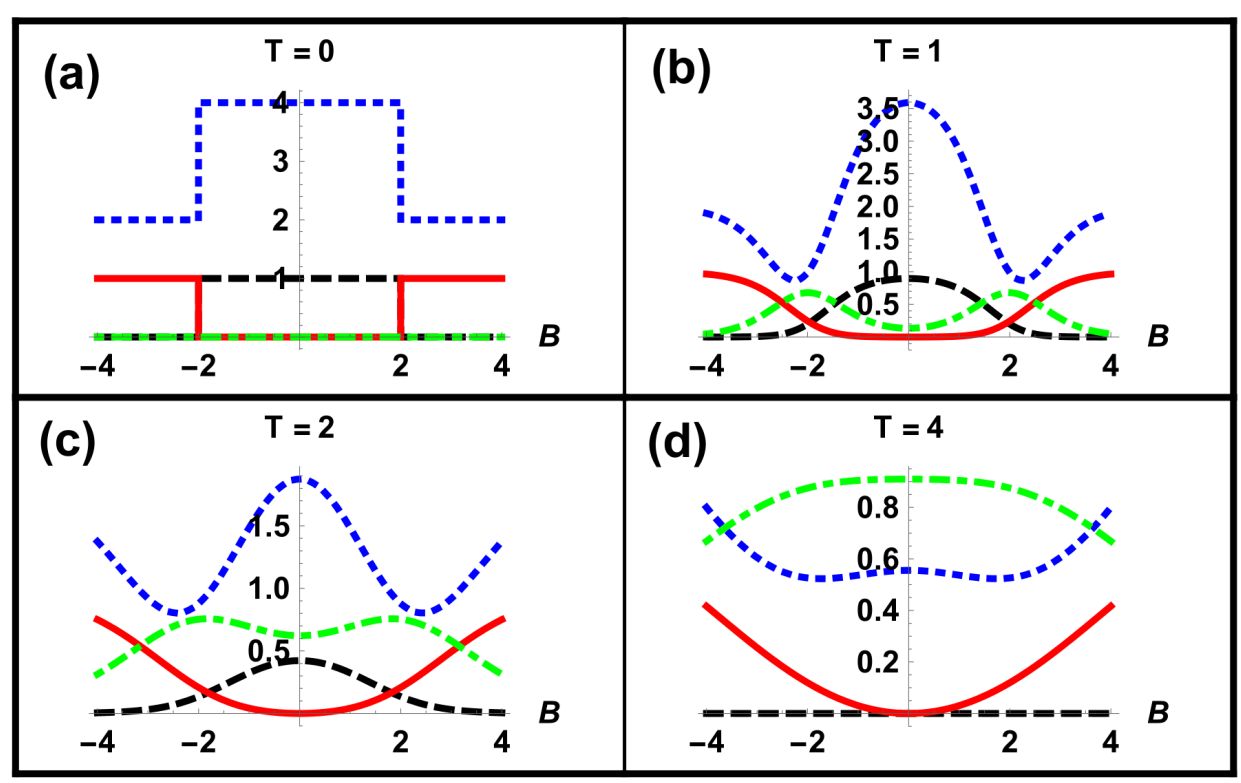

Figure 5. The variation of $F(\rho)$ (dotted, blue), $N(\rho)$ (dashed, black), $F_{o}(\rho)$ (solid, red) and $M(\rho)$ (dotted-dashed, green) versus $B$ for few fixed values of $T$ in antiferromagnetic case

$N(\rho)$. It is also abvious that $N(\rho)$ diminishes to zero earlier than QFI and FOC which shows the fragility of entanglement against bath temperature. Moreoever, It is seen that $M(\rho)$ increases with the increase in $T$ and $B$.

\section{B. Non uniform antiferromagnetic case}

The results of QFI, FOC, QM and Negativity are plotted against $b$ at different fixed values of the $T$ when $J=+1$ and $B=0$ (except for Fig. $7(\mathbf{a})$ where $B=b=0$ ) are shown in Fig. 7. In contrast to uniform antiferromagnetic case, all the quatifiers QFI, Negativity, FOC are single valued functions and so there is no critical point where we can find their non-analytic relation with respect to $b$ and so no quantum phase transition occurs (QPT) in this case. In addition, It is observed that like in earlier discussed Fig. 5 , in this figure again there exist a reciprocal relation in the form of trade off between Negativity and FOC when plotted against $b$ for different fixed values of $T$. The proposed inequalities which shows that there may exist a quantitative relationship between QFI and Negativity for $b$ and low $T$ are also satisfied here as well. Again the equality in the proposed inequalities holds at $T=0$ (Fig. $7(\mathbf{a}))$. Furthermore, again here we can see QFI faithfully behaves with both 


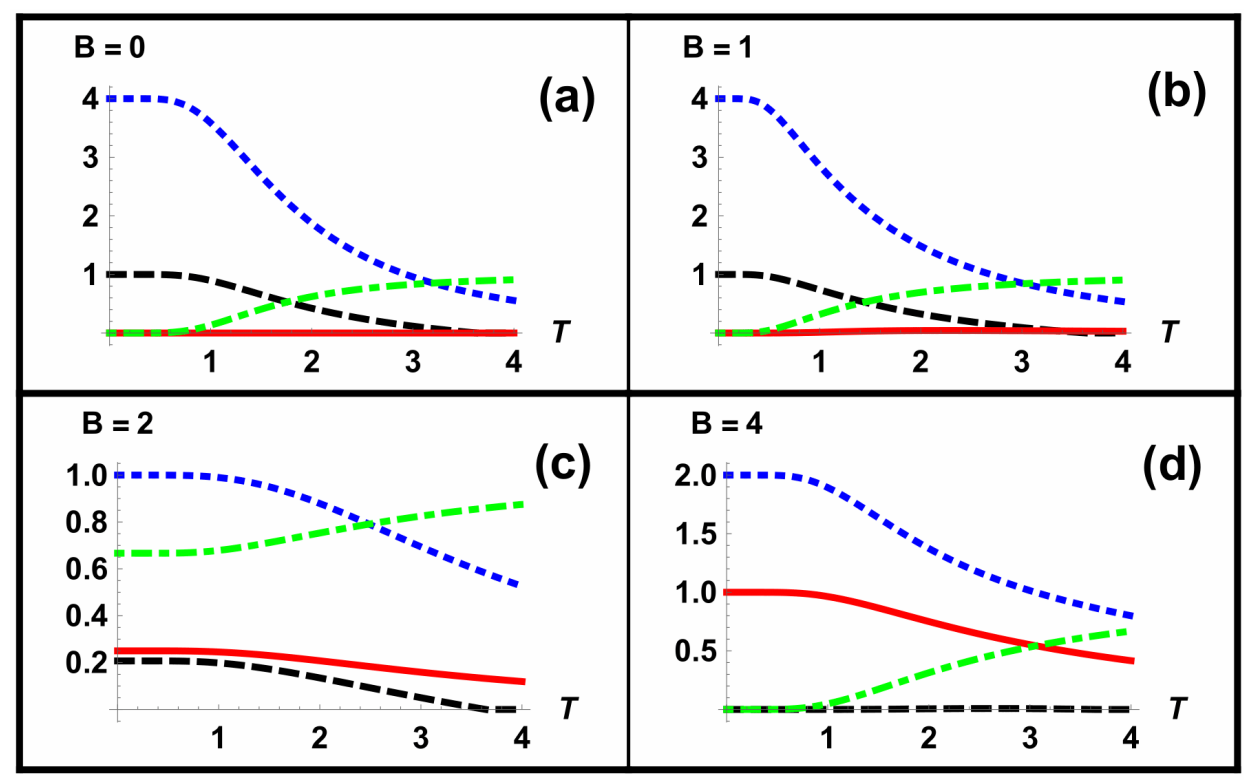

Figure 6. The variation of $F(\rho)$ (dotted, blue), $N(\rho)$ (dashed, black), $F_{o}(\rho)$ (solid, red) and $M(\rho)$ (dotted-dashed, green) versus $T$ for few fixed values of $B$ in antiferromagnetic case

Negativity and FOC. For Instance regions Fig. 7 where Negativity is dominant over FOC quantitatively, there QFI behaves in the same way as Negativity and conversely the regions where FOC is dominant over Negativity QFI favors FOC.

Fig. 8 shows the plot of QFI, Negativity and FOC as a function of $T$ at different fixed values of $b$ when $J=+1$. For $b=0$ in Fig. $8(\mathbf{a})$, it is shown that the ground state is maximally entangled and the so Negativity $N(\rho)=1$. Whereas for different fixed values of $b>0$ peak value of $N(\rho)<1$ which shows that the role of $b$ and $T$ are to diminish $N(\rho)$. It is also abvious that $N(\rho)$ diminishes to zero earlier than QFI and FOC which shows the fragility of entanglement when comes in contact with bath at $T>0$. Moreover, It is seen that QM increases with the increase in $T$ which is trivial as we have calculated the QM for thermal state and in contrast to uniform antiferromagnetic case, in this case increase in the value of $b$ generally slows down the the rate of increase in QM with $T$.

\section{Uniform ferromagnetic case}

Fig. 9 represents the plots of QFI, FOC, QM and Negativity as a function of $B$ at different fixed values of the $T$ when $J=-1$ and $b=0$ (except for Fig. 9(a) in which $B=b=0$ ). In contrast to uniform antiferromagnetic case, It is observed that there is no 


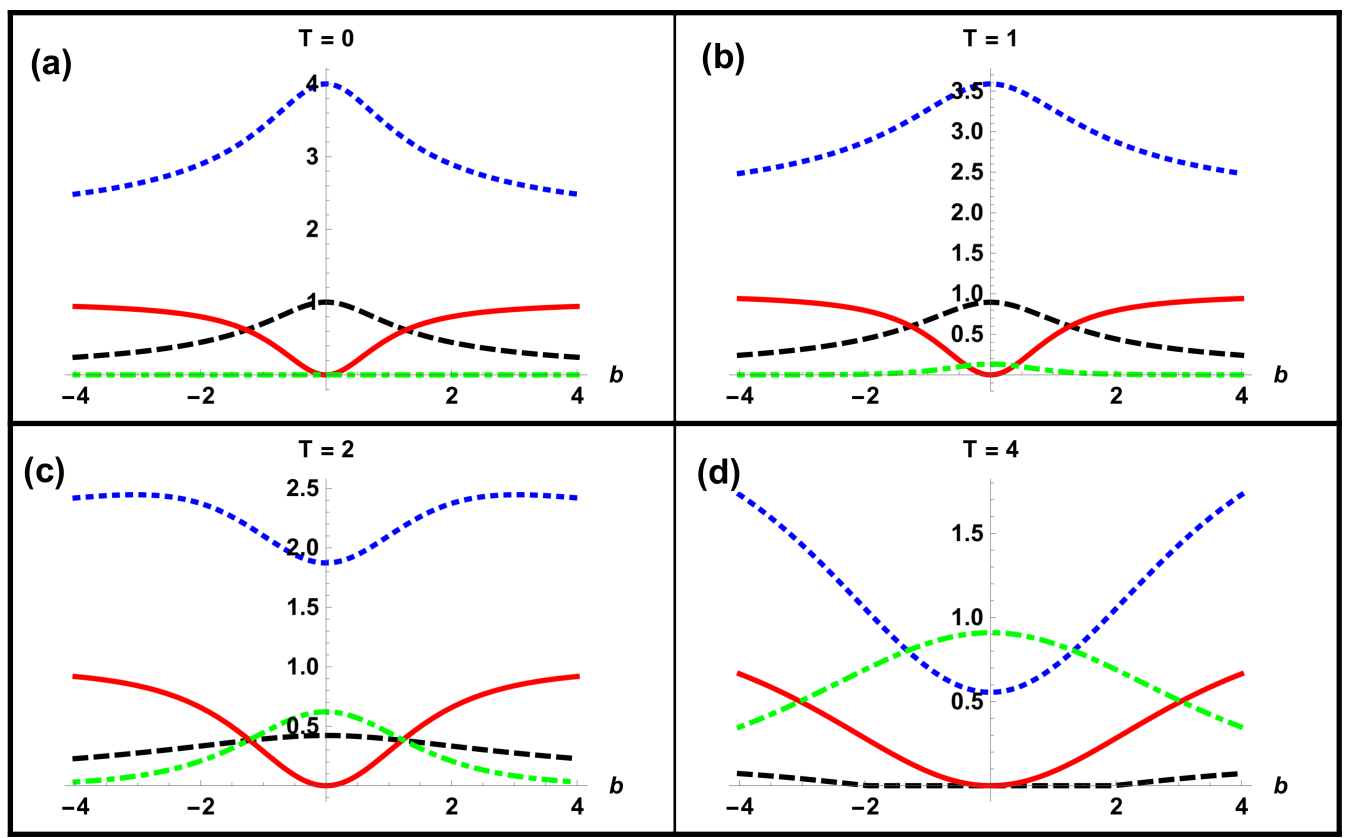

Figure 7. The variation of $F(\rho)$ (dotted, blue), $N(\rho)$ (dashed, black), $F_{o}(\rho)$ (solid, red) and $M(\rho)$ (dotted-dashed, green) versus $b$ for few fixed values of $T$ in non uniform antiferromagnetic case

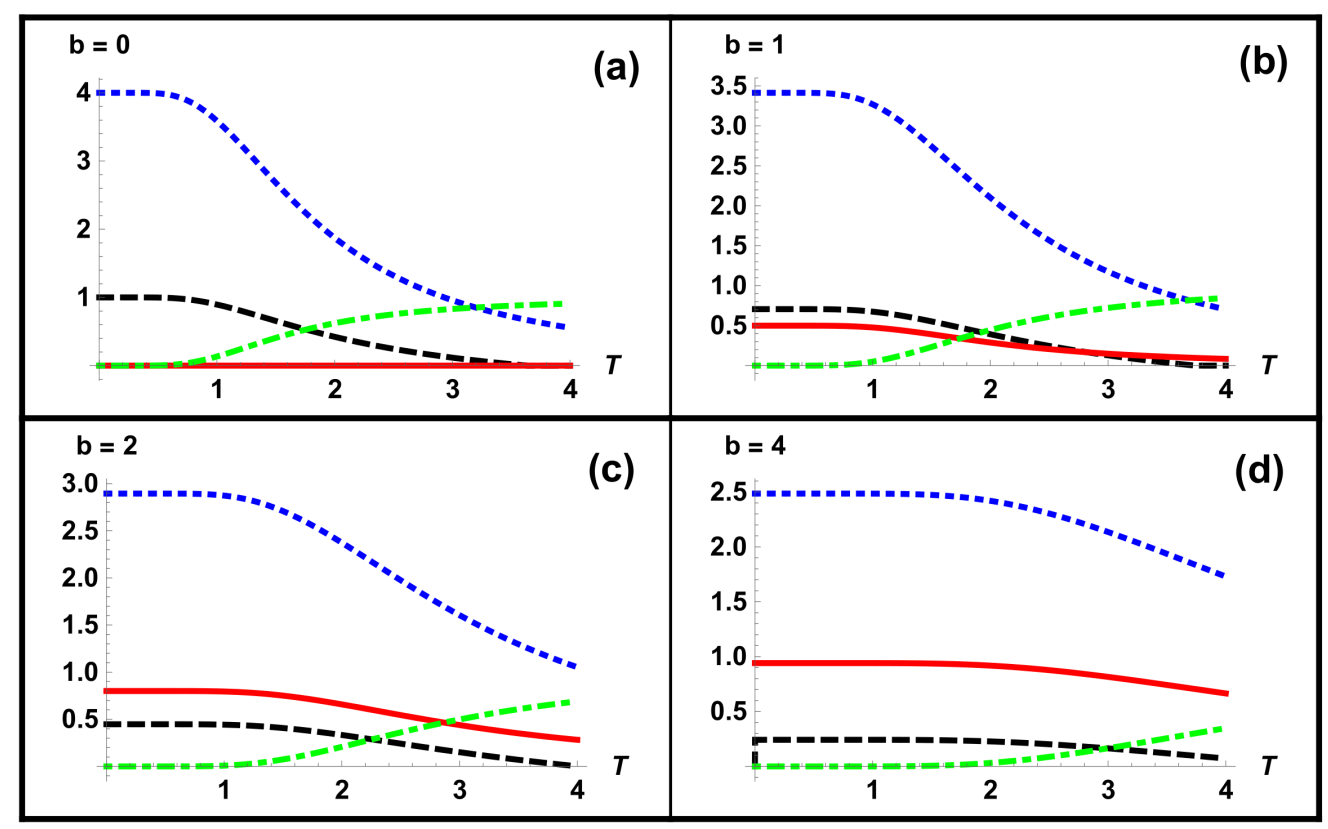

Figure 8. The variation of $F(\rho)$ (dotted, blue), $N(\rho)$ (dashed, black), $F_{o}(\rho)$ (solid, red) and $M(\rho)$ (dotted-dashed, green) versus $T$ for few fixed values of $b$ in antiferromagnetic case

entanglement in this case. In addition, the equality in the proposed inequalities holds at 
$T=0($ Fig. 9(a)). Since there is no entanglement in this case therefore the behavior of QFI is exactly similar to FOC at any value of parameters $B$ and $T$. It is also observed that QM is zero for all the values of $B$ at $T=0$. For small $T$ the behavior of QM is localized at small values of $B$ which becomes delocalize for larger values of $T$.

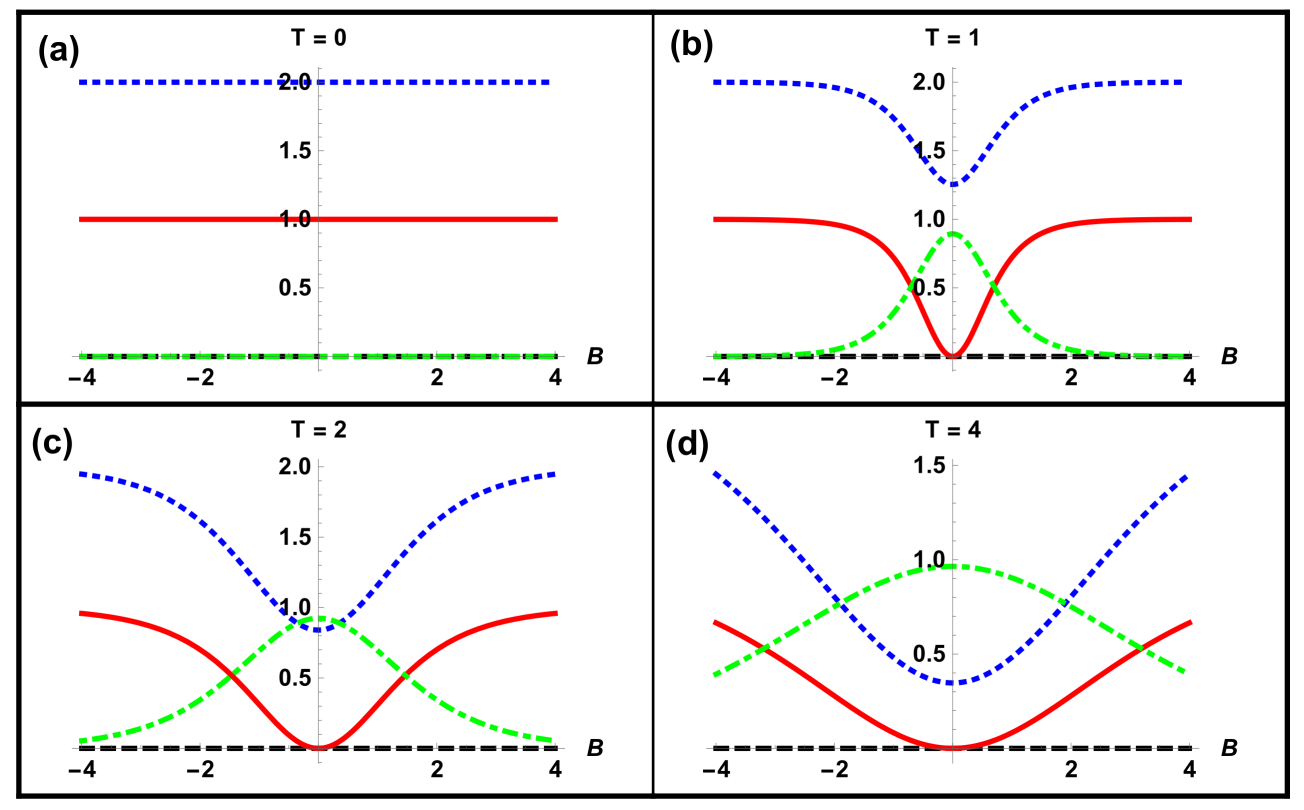

Figure 9. The variation of $F(\rho)$ (dotted, blue), $N(\rho)$ (dashed, black), $F_{o}(\rho)$ (solid, red) and $M(\rho)$ (dotted-dashed, green) versus $B$ for few fixed values of $T$ in ferromagnetic case

Fig. 10 shows the plot of QFI, Negativity and FOC as a function of temperature $T$ at different fixed values of uniform magnetic field $B$ when $J=-1$. For $B=0$ in Fig. 10(a), it is shown that at ground state both FOC and Negativity are zero. Whereas for different fixed values of $|B|>0$ the peak value of FOC is 1 which decreases with temperature. Moreover, the QFI and FOC behaves similar to each other since Negativity is through out zero in this scenario. For smaller $|B|$ FOC decreases faster rate whereas for large $|B|$ its decaying rate with $T$ is low.

\section{Non uniform ferromagnetic case}

Fig. 11 represents variation of QFI, FOC, QM and Negativity as a function of non uniform magnetic field $b$ at different fixed values of temperature $T$ when $J=-1$ and $B=0$ (except for Fig. $\mathbf{9}(\mathbf{a})$ where $b=B=0)$. In contrast to antiferromagnetic case, all 


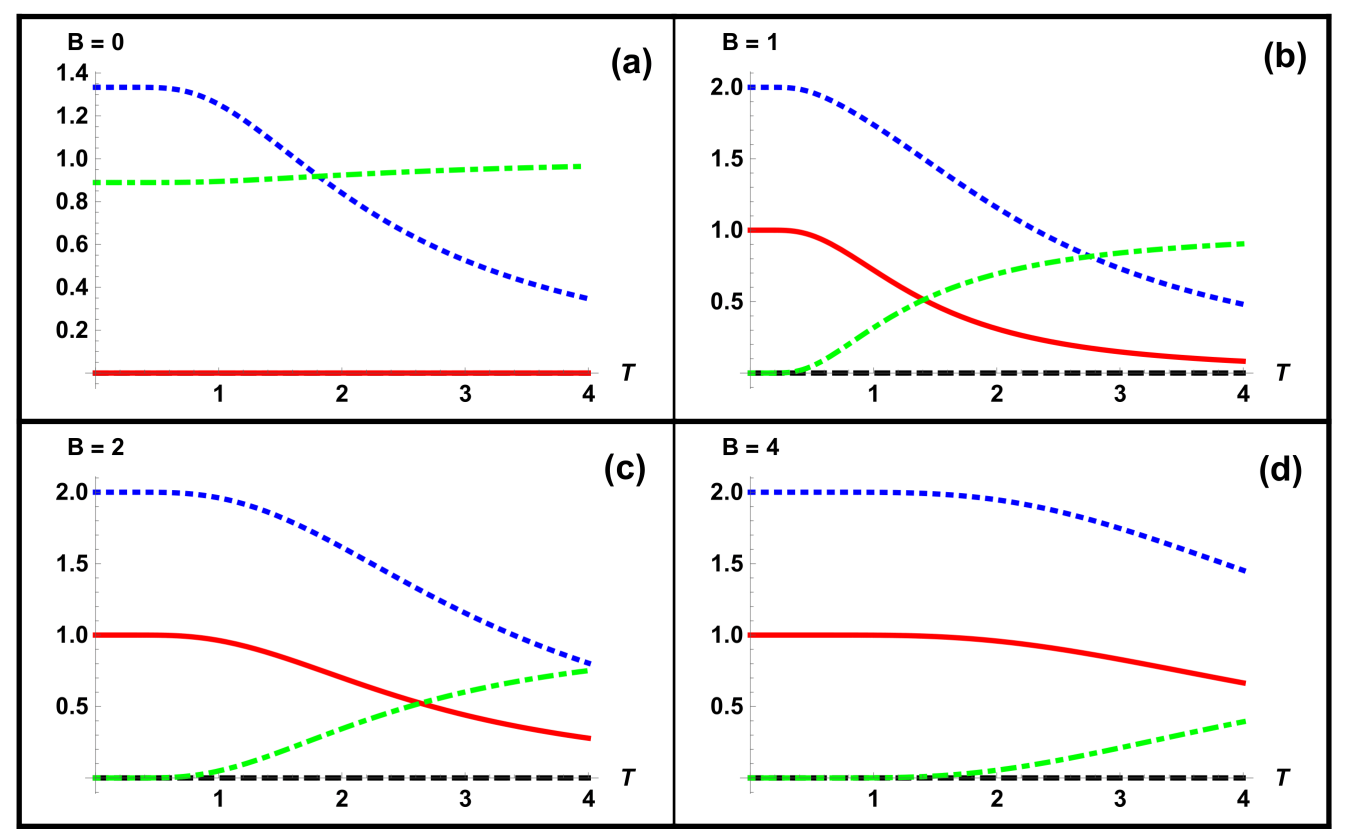

Figure 10. The variation of $F(\rho)$ (dotted, blue), $N(\rho)$ (dashed, black), $F_{o}(\rho)$ (solid, red) and $M(\rho)$ (dotted-dashed, green) versus $T$ for few fixed values of $B$ in uniform ferromagnetic case

the quatifier QFI, Negativity, FOC are well behaved and so there is no critical point where we can find their non-analytic relation with respect to $b$.

In contrast to non uniform ferromagnetic case where is no entanglement at all, in this case entanglement exists which is very sensitive to the values of temperature and non uiform magnetic field $b$. At $T=0$ Negativity is maximum at $b=0$ and start decreasing with $|b|$. Whereas for $T>0$ Negativity is zero at $b=0$ and start increasing after certain value of non uniform magnetic field $b$ means for finite temperature, Negativity start increases and decreases which is unusual. So in this case a very small inhomogeneity is capable of producing large values of thermal entanglement. This shows that the presence of entanglement in the ferromagnetic Heisenberg system is highly unstable against non uniform magnetic fields. Furthermore, this is only case where our proposed inequalities dont hold.

Fig. 12 shows the plot of QFI, Negativity and FOC as a function of $T$ at different fixed values of $b$ when $J=-1$. For $b=0$ in Fig. 10(a), it is shown that at ground state both FOC and Negativity are zero. Whereas for slight increase in $b>0$, FOC and Negativity achieve its peak value (see Fig.12 (b)) which decreases with increase in $T$. 


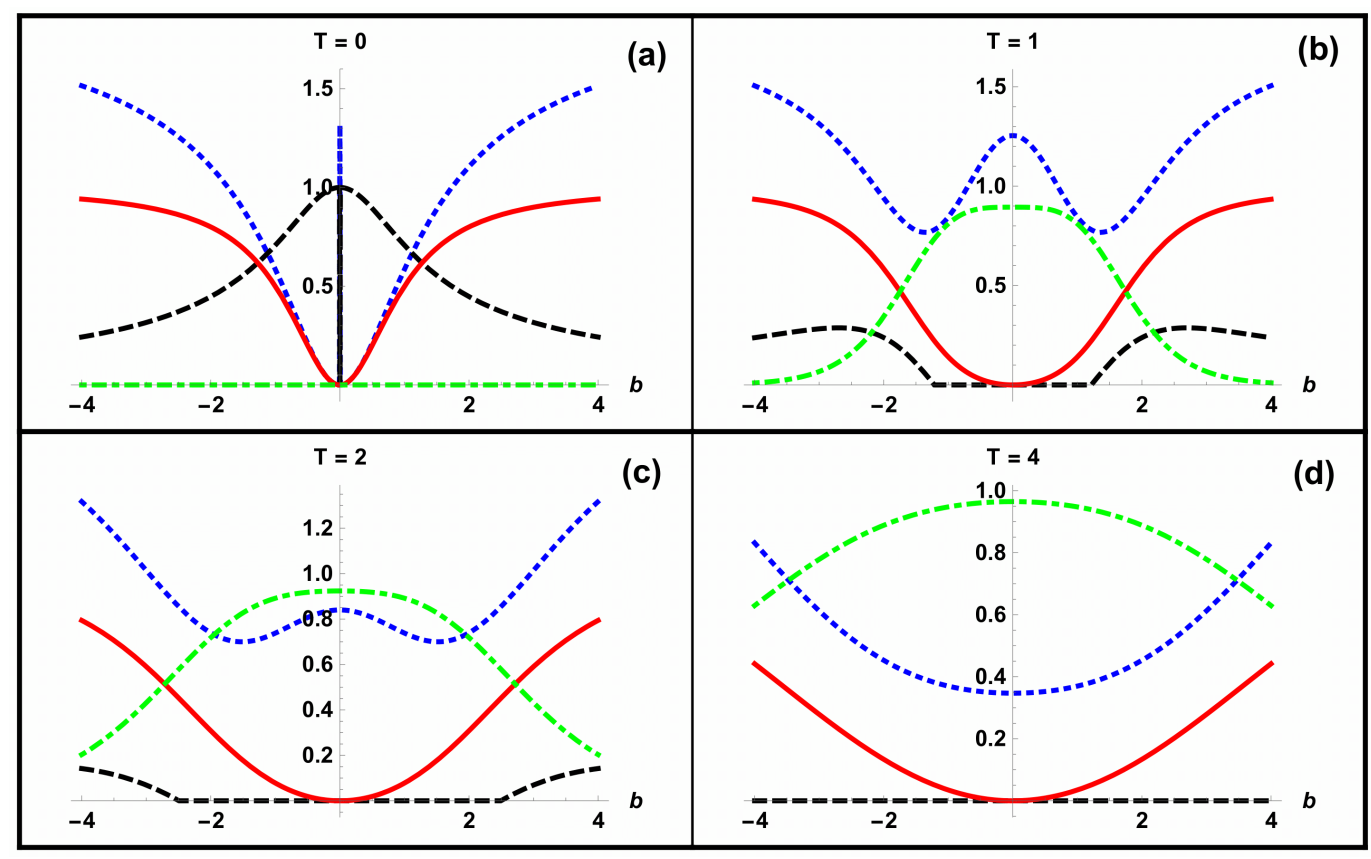

Figure 11. The variation of $F(\rho)$ (dotted, blue), $N(\rho)$ (dashed, black), $F_{o}(\rho)$ (solid, red) and $M(\rho)$ (dotted-dashed, green) versus $b$ for few fixed values of $T$ in non uniform ferromagnetic case

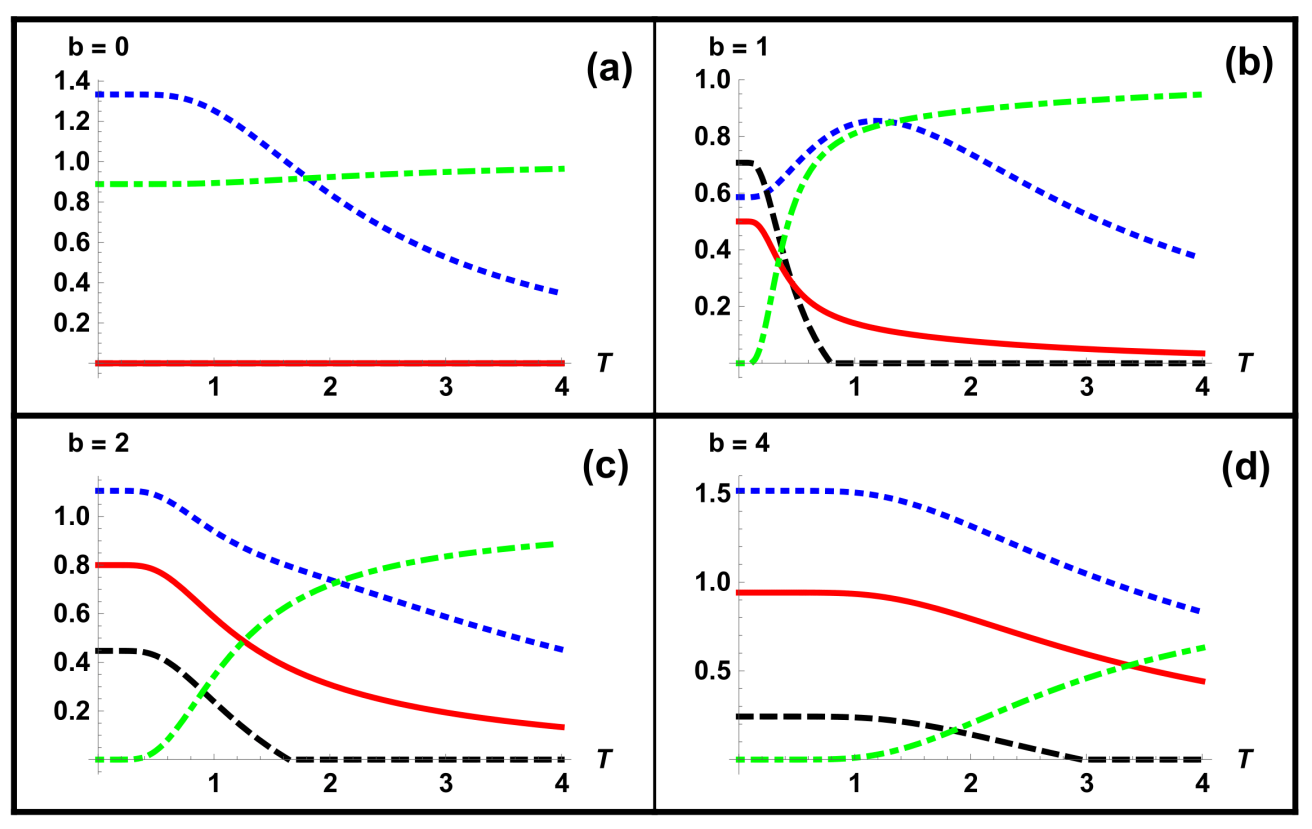

Figure 12. The variation of $F(\rho)$ (dotted, blue), $N(\rho)$ (dashed, black), $F_{o}(\rho)$ (solid, red) and $M(\rho)$ (dotted-dashed, green) versus $T$ for few fixed values of $b$ in non uniform ferromagnetic case 


\section{CONCLUSION}

We have studied the individual as well as collective behavior of thermal quantum coherence, and correlations in two-qubit Heisenberg XXX spin model under independently controllable inhomogeneous magnetic field in terms of FOC, Negativity, and QFI respectively. Generally, it is known that entanglement does not exist in a ferromagnetic spin chain case under a uniform magnetic field, but in our case, we have shown that introducing a little inhomogeneity in a uniform magnetic field to spins creates entanglement in this case which is highly unstable. The strength of this behavior is maximum at $T=0$, and diminishes with $T>0$. We have proposed the inequality relations between QFI and Negativity for our model, that works well for all the situations discussed, except for nonuniform ferromagnetic cases. The reciprocal behavior of FOC and Negativity is also observed in different cases, when plotted against a magnetic field (both uniform and nonuniform). We have realized QFI as a faithful quantifier that captures both entanglement and hidden coherence of individual spins, but it is unable to differentiate among the two when plotted against magnetic field except for the nonuniform ferromagnetic cases.

\section{AUTHORS CONTRIBUTION STATEMENT}

All the authors contributed for the submission of this manuscript in which Asad Ali concieved the idea and perform all the calculation on Mathematica, whereas Mustansar

Nadeem and Professor A. H. Toor and Junaid-ul-Haq discussed the results and Shah Ahad helped in writing the manuscript and editing plots.

\section{AVAILABILITY OF DATA AND MATERIAL}

The data generated for the paper are available upon personal request to the authors.

\section{CODE AVAILABILITY}

The numerical programs are available upon personal request to the authors. 


\section{DECLARATIONS}

\section{Conflict of Interests}

The authors declare that they have no conflict of interest.

[1] Streltsov, A., Adesso, G., \& Plenio, M. B. (2017). Colloquium: Quantum coherence as a resource. Reviews of Modern Physics, 89(4), 041003.

[2] Fanchini, F. F., Pinto, D. D. O. S., \& Adesso, G. (Eds.). (2017). Lectures on General Quantum Correlations and Their Applications (pp. 159-179). Berlin: Springer.

[3] Sun, Y., Mao, Y., \& Luo, S. (2017). From quantum coherence to quantum correlations. EPL (Europhysics Letters), 118(6), 60007.

[4] Scarani, V., Bechmann-Pasquinucci, H., Cerf, N. J., Dušek, M., Lütkenhaus, N., \& Peev, M. (2009). The security of practical quantum key distribution. Reviews of modern physics, 81(3), 1301.

[5] Harrow, A., Hayden, P., \& Leung, D. (2004). Superdense coding of quantum states. Physical review letters, 92(18), 187901.

[6] Gisin, N., Ribordy, G., Tittel, W., \& Zbinden, H. (2002). Quantum cryptography. Reviews of modern physics, 74(1), 145.

[7] Furusawa, A., \& Van Loock, P. (2011). Quantum teleportation and entanglement: a hybrid approach to optical quantum information processing. John Wiley \& Sons.

[8] Horodecki, R., Horodecki, P., Horodecki, M., \& Horodecki, K. (2009). Quantum entanglement. Reviews of modern physics, 81(2), 865 .

[9] Madhok, V., \& Datta, A. (2011). Interpreting quantum discord through quantum state merging. Physical Review A, 83(3), 032323.

[10] Bennett, C. H., DiVincenzo, D. P., Shor, P. W., Smolin, J. A., Terhal, B. M., \& Wootters, W. K. (2001). Remote state preparation. Physical Review Letters, 87(7), 077902.

[11] Simon, D. S., Jaeger, G., \& Sergienko, A. V. (2017). Quantum metrology. In Quantum Metrology, Imaging, and Communication (pp. 91-112). Springer, Cham. 
[12] Petz, D., \& Ghinea, C. (2011). Introduction to quantum Fisher information. In Quantum probability and related topics (pp. 261-281).

[13] Svozilík, J., Vallés, A., Peřina Jr, J., \& Torres, J. P. (2015). Revealing hidden coherence in partially coherent light. Physical review letters, 115(22), 220501.

[14] Radhakrishnan, C., Parthasarathy, M., Jambulingam, S., \& Byrnes, T. (2016). Distribution of quantum coherence in multipartite systems. Physical review letters, 116(15), 150504.

[15] Niezgoda, A., Panfil, M., \& Chwedeńczuk, J. (2020). Quantum correlations in spin chains. Physical Review A, 102(4), 042206.

[16] Cao, M., \& Zhu, S. (2005). Thermal entanglement between alternate qubits of a four-qubit Heisenberg X X chain in a magnetic field. Physical Review A, 71(3), 034311.

[17] Zhang, G. F., \& Li, S. S. (2005). Thermal entanglement in a two-qubit Heisenberg X X Z spin chain under an inhomogeneous magnetic field. Physical Review A, 72(3), 034302.

[18] Wang, X., \& Zanardi, P. (2002). Quantum entanglement and Bell inequalities in Heisenberg spin chains. Physics Letters A, 301(1-2), 1-6.

[19] Parthasarathy, M., Jambulingam, S., Byrnes, T., \& Radhakrishnan, C. (2021). Thermal coherence of the Heisenberg model with Dzyaloshinsky-Moriya interactions in an inhomogenous external field. arXiv preprint arXiv:2103.01130.

[20] Bakmou, L., Slaoui, A., Daoud, M., \& Laamara, R. A. (2019). Quantum Fisher information matrix in Heisenberg XY model. Quantum Information Processing, 18(6), 1-20.

[21] Amazioug, M., Nassik, M., \& Habiballah, N. (2018). Measure of general quantum correlations in optomechanics. International Journal of Quantum Information, 16(05), 1850043.

[22] Yannouleas, C., \& Landman, U. (2007). Symmetry breaking and quantum correlations in finite systems: studies of quantum dots and ultracold Bose gases and related nuclear and chemical methods. Reports on Progress in Physics, 70(12), 2067.

[23] Häusler, W. (1995). Correlations in quantum dots. Zeitschrift für Physik B Condensed Matter, 99(4), 551-560.

[24] Ming, F., Wang, D., Li, L. J., Fan, X. G., Song, X. K., Ye, L., \& Chen, J. L. (2021). Tradeoff Relations in Quantum Resource Theory. Advanced Quantum Technologies, 4(7), 2100036.

[25] Hyllus, P., Laskowski, W., Krischek, R., Schwemmer, C., Wieczorek, W., Weinfurter, H., ... \& Smerzi, A. (2012). Fisher information and multiparticle entanglement. Physical Review A, $85(2), 022321$. 
[26] Qi, X., Gao, T., \& Yan, F. (2017). Measuring coherence with entanglement concurrence. Journal of Physics A: Mathematical and Theoretical, 50(28), 285301.

[27] Liu, X. M., Cheng, W. W., \& Liu, J. M. (2016). Renormalization-group approach to quantum Fisher information in an XY model with staggered Dzyaloshinskii-Moriya interaction. Scientific reports, 6(1), 1-9.

[28] Singh, U., Bera, M. N., Dhar, H. S., \& Pati, A. K. (2015). Maximally coherent mixed states: Complementarity between maximal coherence and mixedness. Physical Review A, $91(5), 052115$.

[29] Vidal, G., \& Werner, R. F. (2002). Computable measure of entanglement. Physical Review A, $65(3), 032314$. 